\title{
Excitation of Intraseasonal Variability in the Equatorial Atmosphere by Yanai Wave Groups via WISHE-Induced Convection
}

\section{Citation}

Solodoch, Aviv, William Boos, Zhiming Kuang, and Eli Tziperman. 2011. "Excitation of Intraseasonal Variability in the Equatorial Atmosphere by Yanai Wave Groups via WISHEInduced Convection." Journal of the Atmospheric Sciences 68 (2) (February): 210-225. doi:10.1175/2010jas3564.1. http://dx.doi.org/10.1175/2010JAS3564.1.

\section{Published Version}

doi:10.1175/2010jas3564.1

\section{Permanent link}

http://nrs.harvard.edu/urn-3:HUL.InstRepos:11891623

\section{Terms of Use}

This article was downloaded from Harvard University's DASH repository, and is made available under the terms and conditions applicable to Other Posted Material, as set forth at http:// nrs.harvard.edu/urn-3:HUL.InstRepos:dash.current.terms-of-use\#LAA

\section{Share Your Story}

The Harvard community has made this article openly available.

Please share how this access benefits you. Submit a story.

Accessibility 


\title{
Excitation of Intraseasonal Variability in the Equatorial Atmosphere by Yanai Wave Groups via WISHE-Induced Convection
}

\author{
Aviv SOLODOCH \\ Weizmann Institute of Science, Rehovot, Israel \\ WiLliam R. BoOs, ZHIMING KUANG, AND ELI TZIPERMAN \\ Department of Earth and Planetary Sciences, and School of Engineering and Applied Sciences, Harvard University, \\ Cambridge, Massachusetts
}

(Manuscript received 25 May 2010, in final form 30 July 2010)

\begin{abstract}
A mechanism is presented, based on multiscale interactions via nonlinear wind-induced surface heat exchange (WISHE), that produces eastward-propagating, intraseasonal convective anomalies in the tropical atmosphere. Simulations of convectively coupled disturbances are presented in two intermediate-complexity atmospheric models. One is a shallow water model with a simple WISHE-motivated heating term. The other model is also based on a first baroclinic mode but has an additional prognostic equation for humidity and a simple representation of moist convection based on a quasi-equilibrium approximation. In spite of many differences between the models, they robustly produce a coherent signal in westerly winds and convection that travels eastward at $4-10 \mathrm{~m} \mathrm{~s}^{-1}$. It is shown here that this slow signal is a forced response to an eastwardpropagating Yanai (mixed Rossby-gravity) wave group. The response takes the form of a forced Kelvin wave that is driven nonlinearly, via WISHE, by meridional wind anomalies of the Yanai wave group and that travels considerably more slowly than the free convectively coupled Kelvin waves in these models. The Yanai waves are destabilized in the models used here by WISHE in the presence of mean easterlies, but more generally they could also be excited by stratiform instability in the absence of mean easterlies so that the mechanism described here could also operate without mean easterlies. Similarities to and differences from the MaddenJulian oscillation (MJO) and convectively coupled tropical waves are discussed.
\end{abstract}

\section{Introduction}

It has been argued that wind-induced surface heat exchange (WISHE) (Emanuel 1987; Neelin et al. 1987), by which ocean evaporation enhanced by surface winds forces atmospheric convection, plays a central role in organizing tropical intraseasonal variability (e.g., Sobel et al. 2008). Emanuel (1987) and Neelin et al. (1987) found unstable Kelvin wave-like solutions due to the coupling of convection, large-scale winds, and surface heat fluxes in linear models, and they suggested that these solutions might explain the dominant pattern of tropical intraseasonal variability: the Madden-Julian oscillation (MJO; Madden and Julian 1971, 1994). While these early

Corresponding author address: William R. Boos, Dept. of Geology and Geophysics, Yale University, P.O. Box 208109, New Haven, CT 06520-8109.

E-mail: billboos@alum.mit.edu solutions have since been recognized to differ fundamentally from the observed MJO (e.g., Zhang 2005; Wang 1988), recent observational and model evidence suggests an important role for WISHE, interpreted more broadly than the specific form used in Emanuel (1987) and Neelin et al. (1987), in tropical intraseasonal variability (Maloney and Sobel 2004; Sobel et al. 2008).

This evidence includes observed relative minima in intraseasonal variance in convection and precipitation over land surfaces in the tropical western Pacific and Indian Oceans, in spite of the high climatological mean levels of convection and precipitation in these regions (Sobel et al. 2008). While this observation could be partially due to nonlinear interactions between intraseasonal convection and the diurnal cycle or to cloudradiative feedbacks, it may also be evidence for the importance of WISHE, which does not typically operate over land because of its low thermal inertia. Other evidence is that significant surface heat flux anomalies occur 
in the active phase of the MJO (Shinoda et al. 1998). In addition, nonlinear WISHE seems to be important in simulated eastward-propagating, intraseasonal disturbances (Raymond 2001; Maloney and Sobel 2004). From a broader and more theoretical point of view, how largescale disturbances in a moist convecting atmosphere behave in the presence of feedbacks from WISHE and other sources of column-integrated moist static energy is a topic of considerable interest. While there have been a number of theoretical studies of the effects of linear WISHE, little has been done in terms of the nonlinear effects of WISHE (Sugiyama 2009a,b). The present paper attempts to explore such nonlinear effects in idealized frameworks.

This paper identifies a novel mechanism in which the equatorial winds due to Yanai [mixed Rossby-gravity (MRG)] wave groups, through nonlinear WISHE, induce enhanced surface heat exchange. The resulting induced convection and atmospheric heating, in turn, force slow eastward-propagating Kelvin waves that occupy the same general region of the wavenumber-frequency spectrum as the observed $\mathrm{MJO}$, at time and space scales longer than those of free convectively coupled Kelvin waves. We will show that this mechanism appears to be at work in two different idealized models.

The model signal presented and analyzed here differs from the observed MJO in some key points, and given these differences and the simplicity of the models, it is not our intention to suggest that this mechanism explains the observed MJO. We nevertheless feel it is an interesting and nontrivial mechanism that can enrich our understanding of tropical dynamics and may be relevant to various convectively coupled disturbances in the tropics and/or in idealized numerical simulations. The correlation of intraseasonal variability and Yanai waves has already been documented by Straub and Kiladis (2003), who also noted that the group velocity of the Yanai waves is similar to the propagation speed of the MJO, a cornerstone of the mechanism proposed here. Our proposed mechanism might be placed in the same general category as that proposed by Majda and Stechmann (2009), in that the primary instability is generated on time and length scales that are short compared to those of the MJO, and there is an upscale transfer of energy to the MJO structure. However, our mechanism is fundamentally different in its focus on Yanai waves as the short time scale agent of instability and in its use of WISHE as the method of energy transfer. The Yanai waves are destabilized in the models used here by WISHE in the presence of mean easterlies (Emanuel 1993), but as discussed in some more detail below they could also be excited by stratiform instability in the absence of mean easterlies (e.g., Mapes 2000; Kuang 2008b).
This work is based on the thesis of the first author [Solodoch 2010; also briefly described in Solodoch et al. (2010)], which used one of the two models described in this paper but explored the behavior of that model and its sensitivity to parameters in greater depth than is presented here. Concurrently with Solodoch (2010) and the present study, a somewhat related idea has recently been presented in a conference abstract (Yang and Ingersoll 2009). Yang and Ingersoll (2011) hypothesize that the MJO may be viewed as a wave packet that consists of Yanai waves. They show that in the National Oceanic and Atmospheric Administration (NOAA) outgoing longwave radiation (OLR) data, there is a correlation between MRG wave activity and the MJO; they also show that in 3D model simulations, independently forced Yanai waves can form wave packets, and the horizontally smoothed variance of these wave packets has features in common with the MJO.

This paper proceeds as follows. The two models are presented in section 2. Their results are presented and compared in section 3 and then analyzed in section 4 to explore the mechanism of the slowly propagating signal. We conclude in section 5 .

\section{The models}

We use two different models of "intermediate" complexity in this study. The first is a shallow water (SW) model, described below and analyzed in greater detail in Solodoch (2010). The second is a model of the tropical atmosphere's first baroclinic mode, with an additional prognostic equation for moisture and a quasi-equilibrium (QE) treatment of moist convection; that model is summarized below and presented in greater detail in an appendix.

\section{a. The shallow water model}

Our objective is to investigate tropical WISHE-related atmospheric intraseasonal phenomena. Here we use a shallow water model on an equatorial beta plane. The model equations are

$$
\begin{aligned}
u_{t}-\beta y v & =-\eta_{x}+\nu \nabla^{2} u \\
v_{t}+\beta y u & =-\eta_{y}+\nu \nabla^{2} v, \quad \text { and } \\
\eta_{t}+H\left(u_{x}+v_{y}\right) & =-Q(u, v, \eta)-\frac{1}{\tau} \eta, \quad \text { where } \\
Q & =\max \left[0, A|\mathbf{v}+\mathbf{U}|\left(\eta-\eta_{\mathrm{sat}}\right)\right] .
\end{aligned}
$$

Equations (1) and (2) are the momentum equations, (3) is the continuity equation, and $Q$ is the total surface heat 
TABLE 1. Standard experiment parameter values.

\begin{tabular}{lll}
\hline \hline Symbol & \multicolumn{1}{c}{ Value } & \multicolumn{1}{c}{ Description } \\
\hline$H$ & $50 \mathrm{~m}$ & Equivalent depth \\
$\nu$ & $3 \times 10^{5} \mathrm{~m}^{2} \mathrm{~s}^{-1}$ & Eddy viscosity \\
$\tau$ & 5 days & Newtonian cooling time \\
$A$ & $2 \times 10^{-6} \mathrm{~m}^{-1}$ & WISHE coefficient \\
$\eta_{\text {sat }}$ & $-500 \mathrm{~Pa}$ & Saturation pressure \\
$U$ & $-2 \mathrm{~m} \mathrm{~s}^{-1}$ & Mean zonal wind \\
\hline
\end{tabular}

flux. The zonal and meridional velocities are $u$ and $v$, respectively, and $\eta$ is the perturbation (hydrostatic) pressure normalized by the density. The equivalent depth is $H$ (converted to pascals), $\nu$ is the eddy diffusivity, and $\tau$ is the Newtonian cooling time scale. Since we are interested in zonally propagating dynamics, the model is periodic in the east-west coordinate $x$. Parameter values and other specifications are given in Table 1.

The SW model can be viewed as a model of the single vertical mode approximation of the free troposphere temperature, or equivalently from the quasi-equilibrium view, as a model of the subcloud-layer moist static energy. We shall discuss the model in terms of the free troposphere temperature; descriptions in terms of the subcloud-layer moist static energy will be analogous. Observations show that $H=10-60 \mathrm{~m}$ in convectively coupled waves in the tropics (Wheeler and Kiladis 1999). Since the processes that set this equivalent depth (e.g., Mapes 2000; Kuang 2008b) are absent in this model, we simply set the equivalent depth of the model to be $H=50 \mathrm{~m}$, based on the observed values. This equivalent depth is smaller than the dry value for a single vertical mode approximation of the free troposphere temperature because adiabatic cooling due to large-scale ascent is partially compensated by convective heating, so vertical motion in the tropical atmosphere experiences a reduced effective static stability.

The model is forced by a heating term in the mass conservation equation (Gill 1980), represented by a velocitydependent, nonlinear bulk surface flux formula. This surface flux is felt throughout the troposphere by the action of convection, and the divergent flow it drives also feels the reduced effective static stability. Here $\eta_{\text {sat }}$ represents an atmosphere in equilibrium with the surface, while $\eta=0$ represents the state of radiative equilibrium. In steady state and without any divergent flow, the continuity equation simply describes the state of radiative-convective equilibrium, with surface heat fluxes (constrained here to be positive) balancing radiative cooling. One may also view (3) as an equation for the columnintegrated moist static energy assuming a constant relative humidity. In this view, $H$ also describes the gross moist stability, which with a constant relative humidity is proportional to the effective static stability in a moist convective atmosphere. Such equivalence was discussed, for example, in Neelin and Yu (1994) and Emanuel et al. (1994).

In the standard experiment, mean surface easterlies, $U<0$, which prevail over the equatorial oceans, are prescribed in the WISHE term by setting $\mathbf{U}=-|U| \hat{\mathbf{x}}$ in (4). This mean wind is prescribed only in the forcing term in the shallow water model, while the quasi-equilibrium model described below includes the mean advection in its equations as well. It is recognized that observed intraseasonal variability, and that of the MJO in particular, occurs in some regions with mean westerlies and that our use of mean easterlies may seem reminiscent of earlier models for linear WISHE (e.g., Emanuel 1987; Neelin et al.1987). However, the role of mean easterlies in our model is primarily to destabilize Yanai waves. If strong Yanai wave groups could be produced by some other process not included in the models we use here, such as stratiform instability (Mapes 2000; Kuang 2008b), then our mechanism might operate even in the absence of mean easterlies. This is further discussed in section $4 \mathrm{e}$ below.

The mass source $Q$ serves as an analog for winddependent surface heat fluxes over the tropical ocean, with $A$ being an exchange coefficient (which includes some of the scalings needed to allow $\eta$ to serve as an analog for observed thermodynamic quantities, such as boundary layer moisture or tropospheric thickness) and $\eta_{\text {sat }}$ a spatially and temporally invariant "saturation" perturbation pressure. Note that the positive-definite $Q$ represents only the portion of convective heating that varies in response to surface heat fluxes, with the typically larger portion that balances adiabatic cooling implicitly included by our choice of equivalent depth. We have chosen $\eta_{\text {sat }}$ to be negative, so that a mass sink in this model is the analog of convective heating. The above system, which is henceforth referred to as the shallow water model, might thus be physically interpreted as a model for the atmospheric boundary layer that is vented by deep convective mass fluxes (Lindzen and Nigam 1987), or alternatively it may be viewed as a variant of the Gill (1980) model.

The numerical model used to represent this system is the Massachusetts Institute of Technology general circulation model (MITgcm) (Marshall et al. 1997a,b) run in its atmospheric shallow water mode. The forcing is added to the continuity equation via the freshwater model forcing option. The domain consists of $128 \times 64$ $(x, y)$ square grid cells, each $200^{2} \mathrm{~km}^{2}$ in area. Integration is done with a time step of $450 \mathrm{~s}$. Zonally periodic boundary conditions and no-normal-flow, no-slip meridional boundary conditions are applied, along with a sponge layer 

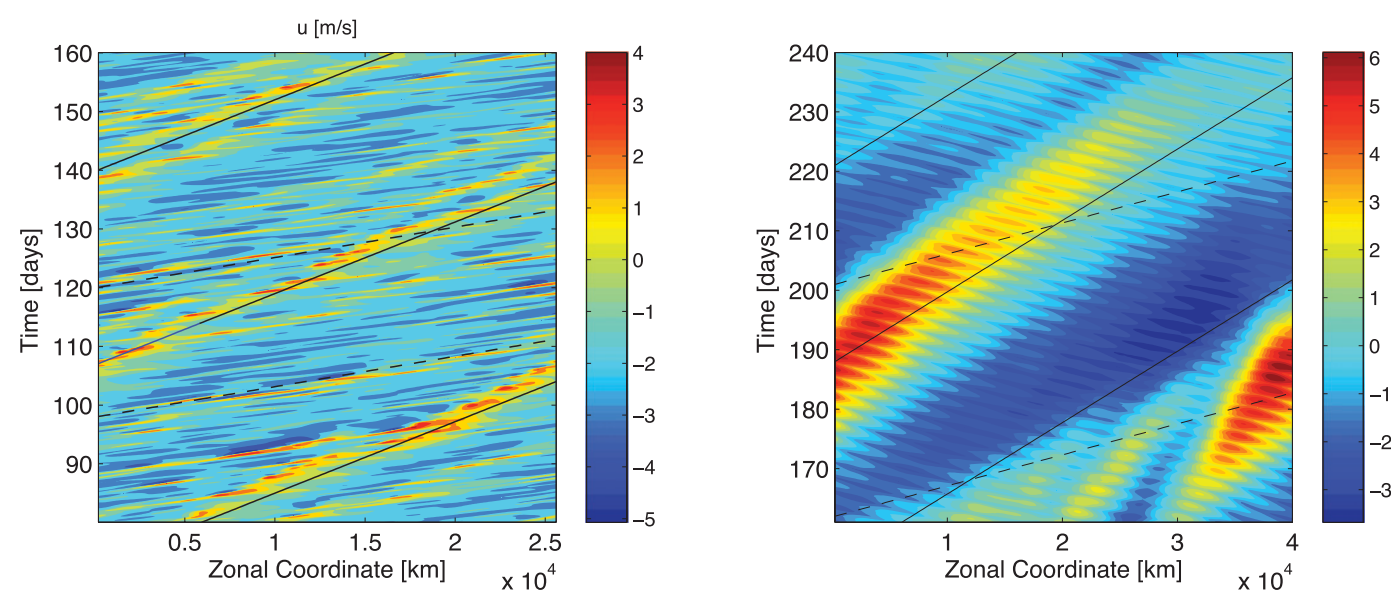

FIG. 1. Hovmöller diagrams of $u\left(\mathrm{~m} \mathrm{~s}^{-1}\right)$ at the equator: (left) SW and (right) QE models. The dashed lines denote the free Kelvin wave speed of $22 \mathrm{~m} \mathrm{~s}^{-1}$. The solid black line denotes a fit to the slow signal propagation speed of $9.5 \mathrm{~m} \mathrm{~s}^{-1}$.

on the meridional boundaries. The model converges to the variability shown below regardless of the initial perturbation used to initialize it.

\section{b. The quasi-equilibrium model}

We also employ a model that is dynamically similar to that discussed above, in that it represents a single baroclinic mode of the tropical free troposphere, but that employs an additional prognostic equation for the humidity of the tropical boundary layer. It also incorporates a convective mass flux that couples this boundary layer humidity with free-tropospheric temperature (or, equivalently, with free-tropospheric thickness), and a closure that relaxes this mass flux toward the value needed to keep convective available potential energy (CAPE) in quasi-equilibrium. This system is similar to the analytical model described by Emanuel (1993), but is solved here numerically; an axisymmetric version with the addition of a barotropic mode was described in Boos and Emanuel (2008). The model is also structurally similar to the quasiequilibrium tropical circulation model of Neelin and Zeng (2000) but lacks a barotropic mode. It is henceforth referred to as the QE model.

To obtain the slow signal in this model, the parameter $\chi$, which controls the drying of the subcloud layer by convective downdrafts, was set to a low value so that the model operated near a high-humidity limit (subcloud relative humidities were typically near $95 \%$ ). Given the highly idealized nature of this model and the number of processes omitted from this model (e.g., the dependence of radiative cooling on moisture), the model may have relevance to the observed atmosphere even while operating near this limit. But even if it is irrelevant, the multiscale, nonlinear WISHE phenomenon it produces seems to be a theoretical problem worth exploring.

\section{Model results}

Both models yield intriguing slow, eastward-propagating signals seen in Hovmöller diagrams of the zonal velocity at the equator (Fig. 1). The dashed diagonal lines on the plots show the free Kelvin wave propagation speed $\left(22 \mathrm{~m} \mathrm{~s}^{-1}\right)$, which is clearly much faster than the speed of the slow signal denoted by the solid lines $\left(9.5 \mathrm{~m} \mathrm{~s}^{-1}\right)$. The propagation speed varies slightly in time in both models, indicating that the slow signal is a wideband phenomenon. In the QE model a low wavenumber signal sometimes propagates at a speed closer to $5 \mathrm{~m} \mathrm{~s}^{-1}$ (e.g., bottom right of the QE model panel in Fig. 1). The shallow water model includes significant power at additional frequencies and propagation speeds, but in both models it is clear that the propagating signal is an envelope encompassing faster phase propagation. This is even more evident in the Hovmöller diagrams of the meridional velocities in Fig. 2.

We will show in the next section that the slow eastwardpropagating signal seen in the $u$ Hovmöller plots is a forced Kelvin wave distinct from the models' free Kelvin waves, and that the faster signals, seen in the $v$ Hovmöller plots in particular, are due to Yanai waves. We will further show that the group velocity of the Yanai waves is equal to the propagation speed of the slowly propagating signal, and that the Yanai waves nonlinearly drive the slow signal via WISHE.

A significant difference between the two models is the structure of the phase propagation seen within the slowly propagating signal. In the shallow water model (left panel in Fig. 1) the slow signal consists of an envelope of short-lived Kelvin waves that are continuously excited with 1- to 2-day intervals and typically start a few hundred kilometers to the west of the slow signal. In the 

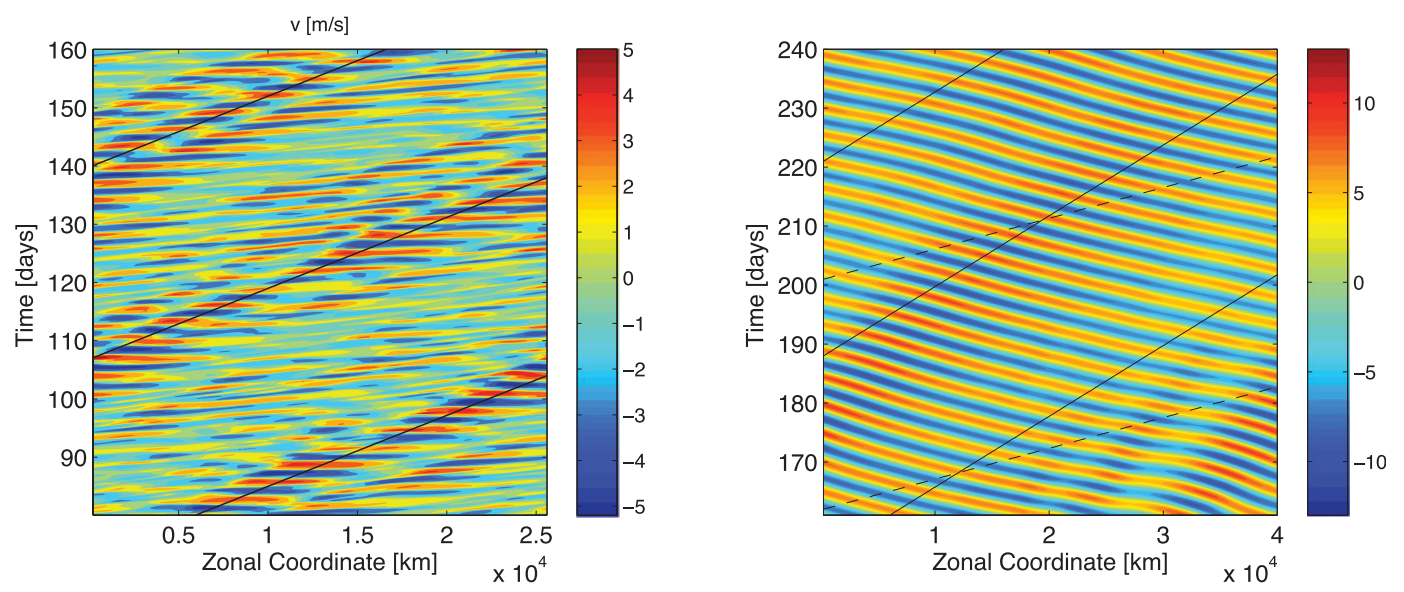

FIG. 2. Hovmöller diagrams of $v\left(\mathrm{~m} \mathrm{~s}^{-1}\right)$ at the equator for the (left) SW and (right) QE models. The solid black line denotes a fit to the slow signal propagation speed of $9.5 \mathrm{~m} \mathrm{~s}^{-1}$.

QE model, the phase propagation is almost exclusively westward in the $u$ Hovmöller as well as in the $v$ Hovmöller. In contrast, the $v$ Hovmöller for the shallow water model shows faster phase propagation that is both easterly and westerly (Fig. 2).

Projecting the $v$ field on equatorial modes (see Solodoch 2010), it is found that Yanai waves, whose meridional velocity has a Gaussian structure in latitude that peaks at the equator, comprise over $70 \%$ of the $v$ variability near the equator in the shallow water model. This fact will later simplify our analysis of their interaction with the slow signal.

Power spectra as a function of zonal wavenumber $k$ and frequency $\omega$ are shown in Figs. 3 and 4 for $u$ and $v$, respectively. Theoretical dispersion curves of equatorial waves are superimposed over these power spectra, and much of the spectral power is concentrated near these dispersion curves, indicating that the dynamics are not very far from the linear regime. However, harmonics in the spectra of both models, which seem more prominent in the QE model, do suggest that nonlinear interactions occur. Indeed, the mechanism proposed below for the generation of the slow signal is a nonlinear interaction, occurring via WISHE. Clear and distinct spectral peaks are associated with linear Yanai waves in both models, but there is no enhanced power in Rossby wave modes. The spectrum of $u$ for the QE model has a pronounced minimum at $k=0$ due to the absence of any variability of zonal mean $u$ precisely on the equator in that model; this minimum is much less pronounced when power is summed over several degrees of latitude surrounding the equator (not shown).

Our main focus in this paper is the interesting feature in the $k-\omega$ spectra of the $u$ field (Fig. 3) in the region just below the linear Kelvin wave dispersion line, which is not associated with any linear wave modes. This feature does not appear in the $v$ spectra, is prominent at the equator, and is near linear in $k-\omega$ space. These are all Kelvin wave-like characteristics, as is the fact that this feature appears similarly in the $\eta$ spectrum (not shown). This "sub-Kelvin" feature has its peak energy near the $k=1$ planetary scale, and its energy generally decreases with $k$. The speeds of this sub-Kelvin feature are found from the $k-\omega$ spectrum to be in the range $4-12 \mathrm{~m} \mathrm{~s}^{-1}$, so this feature simply corresponds to the slow, eastwardpropagating signal seen in the Hovmöller diagrams discussed above. Note that near $k=5$ the peak power of this sub-Kelvin feature in the QE model lies at a phase speed near one-quarter of the free Kelvin wave speed, whereas in the shallow water model it is closer to one-half the free Kelvin wave speed.

In the shallow water model, the slow sub-Kelvin feature in the spectrum accounts for $50 \%$ of the zonal wind variability at the equator (Solodoch 2010). An $x-y$ plot of the dynamical fields composited over several realizations of the propagating signal (Figs. 5 and 6) shows that the dynamical structure is similar to that of a Kelvin wave: $u$ and $\eta$ are in phase and rapidly decrease away from the equator, while $v$ is much weaker.

The shallow water model also has significant energy in the first few equatorial modes of inertia-gravity waves at higher wavenumbers (around $k=13$, not shown here; see Solodoch 2010). These disturbances are prominent at higher latitudes, as seen in snapshots of zonal velocity (Fig. 7). Qualitatively similar but weaker variability occurs in the QE model, but in that model the existence of these extratropical, high-wavenumber disturbances is found to be sensitive to model resolution and to the specified convective lag time. The slow signal in the QE model persists even when values of those parameters are 

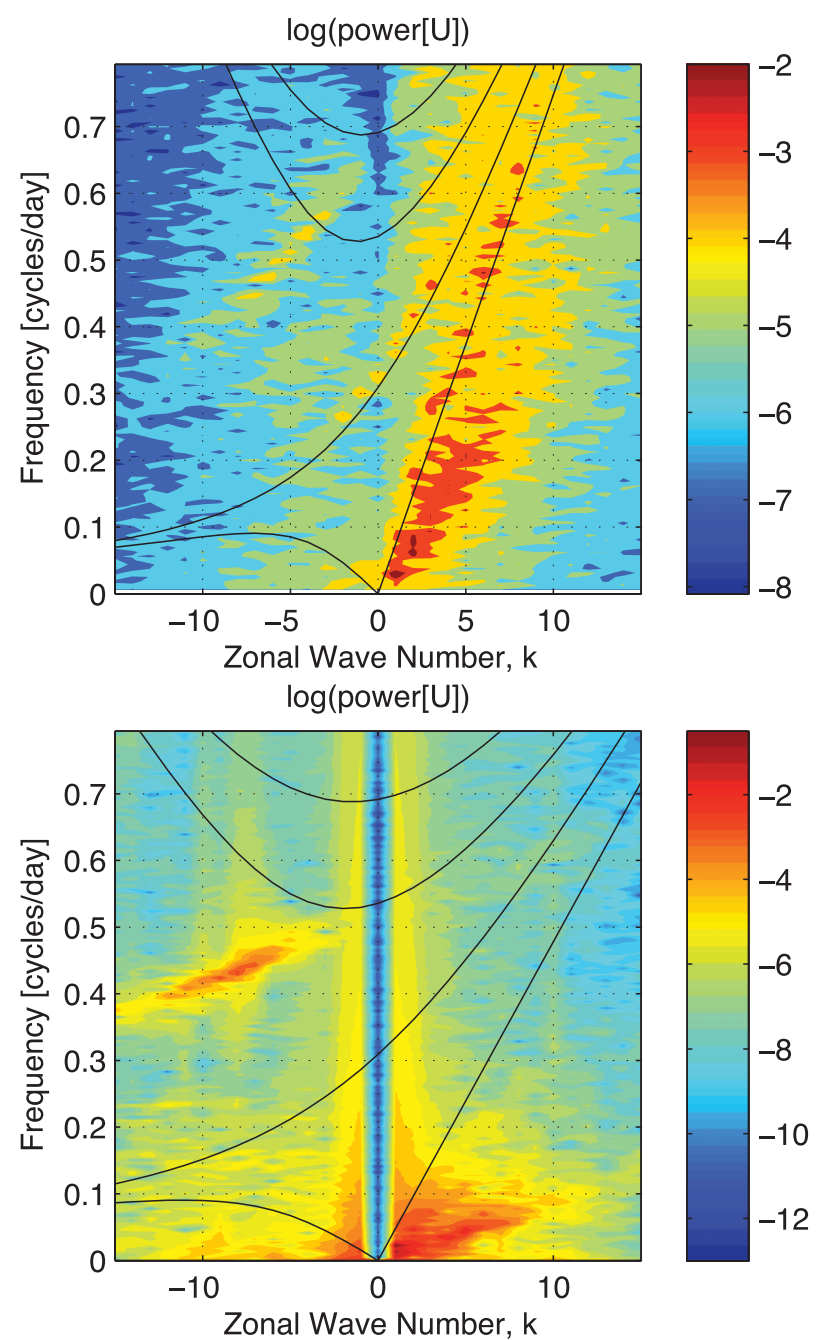

FIG. 3. Spectral power of equatorial $u$, in base-10 log scale, for the (top) SW and (bottom) QE models. Power is summed over the two latitude grid points straddling the equator for the SW model and is for the single grid point precisely on the equator for the QE model. Dispersion curves of equatorial waves are plotted on top of the spectral power. In the SW model, $k$ is defined with respect to the domain width of that model, which is $25600 \mathrm{~km}$.

chosen that eliminate these extratropical disturbances, which shows that they are not an essential part of the slow signal dynamics that are of interest here.

\section{The proposed mechanism}

\section{a. Overview}

To summarize the main results of the previous section, a prominent slow, eastward-propagating signal in both models was found in near-equatorial $u$ and $\eta$ but was notably absent in $v$ (Fig. 1). Its propagation speed was 4-12 $\mathrm{m} \mathrm{s}^{-1}$, considerably slower than the models' free Kelvin wave speeds. The slow signal has numerous Kelvin
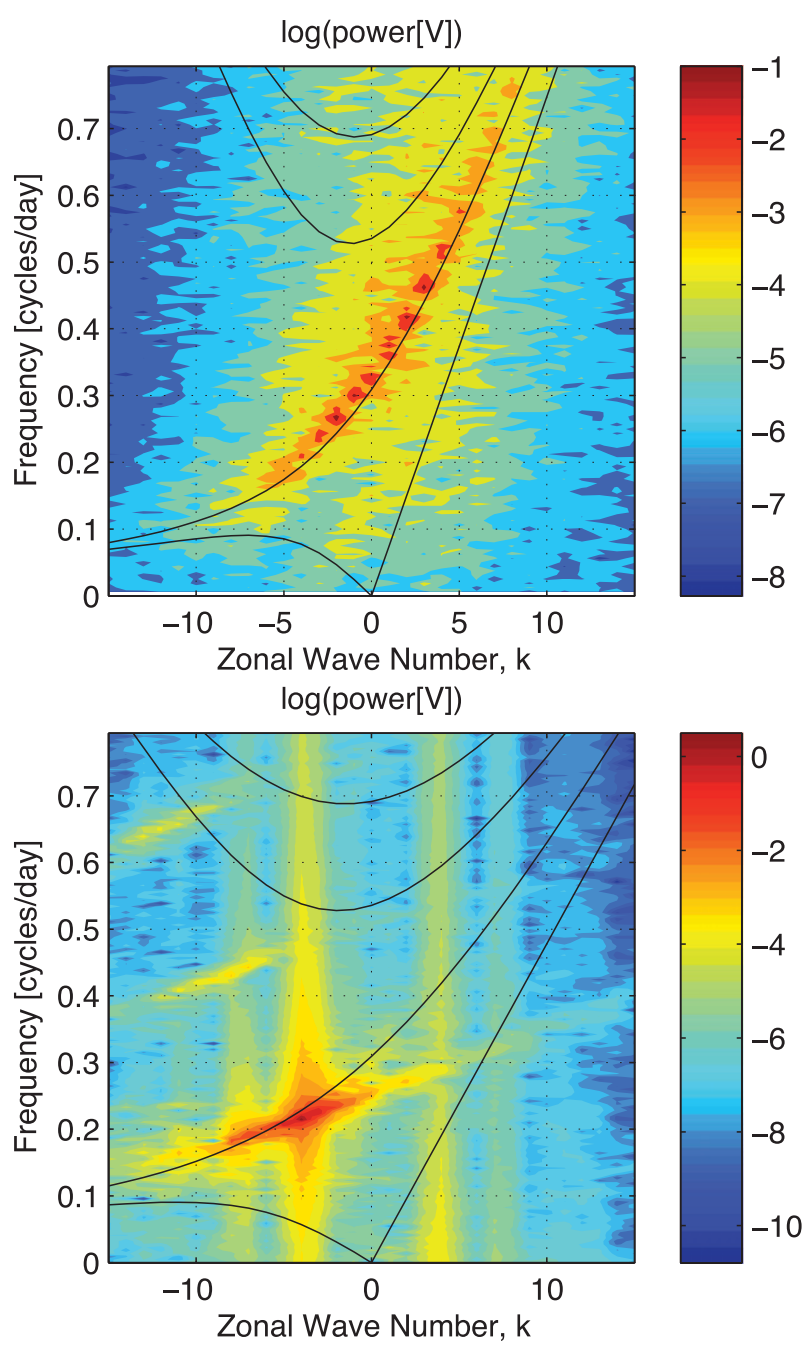

FIG. 4. As in Fig. 3, but for the $v$ velocity field.

wave-like characteristics and involves modulated higherfrequency variability consisting of both Yanai waves and faster free Kelvin waves. The variance of the slow signal peaks at the planetary scale in the zonal direction.

In this section we will show that the slow, eastwardpropagating signal is a Kelvin wave forced by a Yanai wave group, with the forcing occurring through the model terms that represent wind-dependent surface heat fluxes. Our analysis will proceed as follows. The Yanai waves are expected to influence equatorial surface heat fluxes primarily through their $v$ velocity because it is large compared to their equatorial $u$ and $\eta$ anomalies. A Taylor expansion of the bulk formula for surface heat exchange will be used to show that the largest term dependent on meridional wind is proportional to $v^{2}$. The quantity $v^{2}$, being an energy-like property of a wave group, propagates eastward at the Yanai group velocity, producing surface fluxes that in turn force Kelvin waves. Note that the response we 

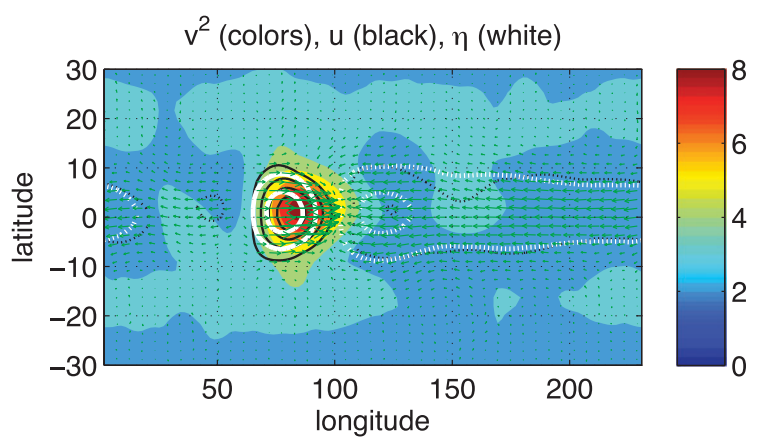

Heating (colors), u (black), $\eta$ (white)
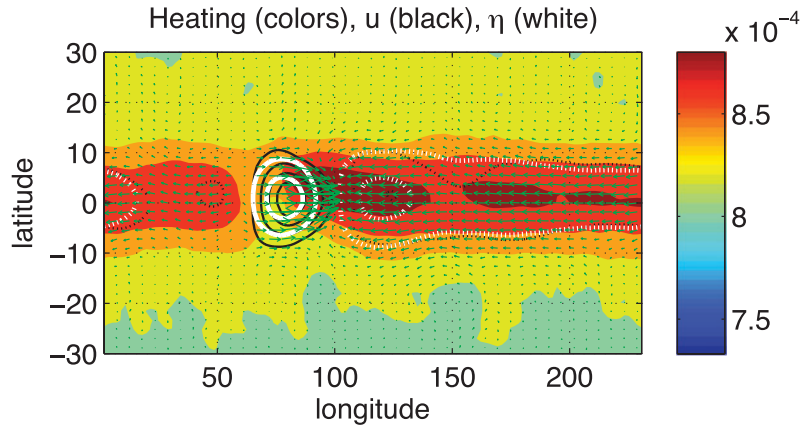

FIG. 5. Composites of the slow signal from the SW model: (top) $v^{2}, u$, and $\eta$ and (bottom) $-Q, u$, and $\eta$. Vectors denote the horizontal wind field.

are suggesting is a forced, damped Kelvin wave that travels at the speed of the Yanai group forcing, and is distinct from the free Kelvin waves that have phase speeds and meridional length scales set by the models' equivalent depths. Indeed, we show below that the slow propagation speed of the forced Kelvin signal is consistent with the shorter meridional length scale of that signal.

\section{b. Analysis}

We begin by noting the existence of a strong spectrum of Yanai waves in both models. The destabilization of these waves by the WISHE feedback was demonstrated in the presence of background easterlies by Emanuel (1993), who also showed that a nonzero convective delay can strongly damp eastward-propagating Yanai waves so that westward-propagating Yanai waves are most unstable. This scale-selective damping occurs because a convective delay biases the convective heating into the cold part of the wave, and the bias creates a stronger anticorrelation between temperature and heating for higher-frequency waves. The preferential damping of higher-frequency waves is consistent with the fact that only westward propagation of the Yanai $v$ signal was seen in the QE model, which employs a nonzero convective delay, while the shallow water model produces both eastward and westward Yanai waves (Fig. 2). More importantly, the westward-propagating Yanai waves excited in the presence of the convective delay in the QE
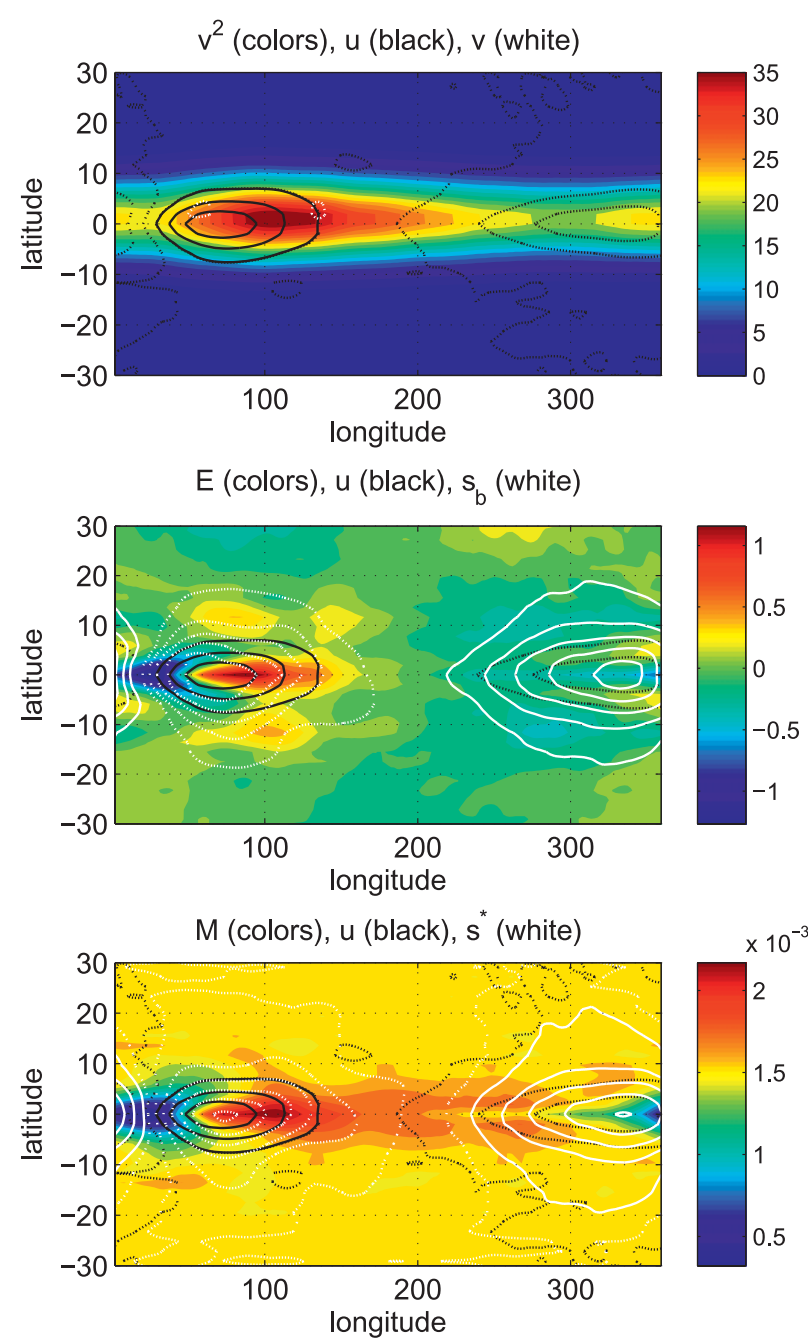

FIG. 6. Composites of the slow signal from the QE model: (top) $v^{2}, u$, and $v$; (middle) surface evaporation $E, u$, and subcloud entropy $s_{b}$ (note that zonal mean $E$ was subtracted to better illustrate the response); and (bottom) convective mass flux $M, u$, and $s^{*}$.

model have a slower group propagation, leading to the excitation of a significantly slower forced Kelvin wave than in the SW model.

Consideration of the meridional structure of the Yanai wave group and its induced heating will prove central to our proposed mechanism. Standard solutions (e.g., Gill 1982) for linear, equatorially trapped shallow water waves give the meridional structure of individual Yanai waves:

$$
\left(v_{Y}, u_{Y}, \eta_{Y}\right) \propto\left(1,-\frac{\omega y}{c_{0}},-\frac{\omega y}{g}\right) \exp \left(\frac{-y^{2}}{2 R_{0}^{2}}\right) .
$$

Individual Yanai waves thus have an equatorially symmetric and wavenumber-independent Gaussian meridional structure in $v_{Y}$, while their meridional structure in $u_{Y}$ and 
u

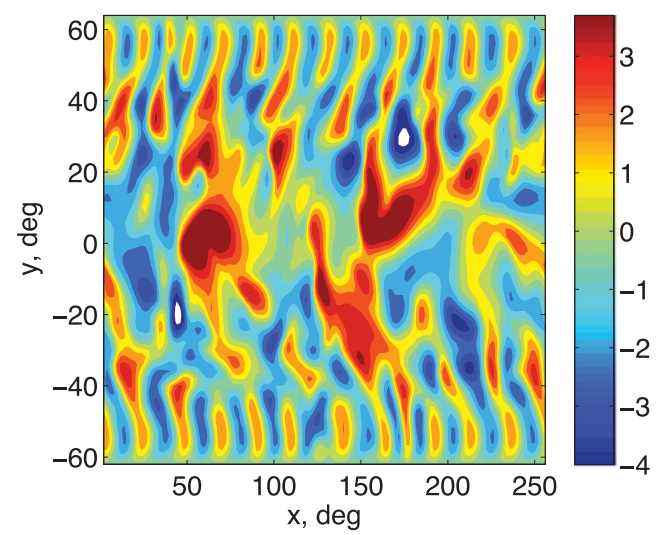

$\mathrm{u}$

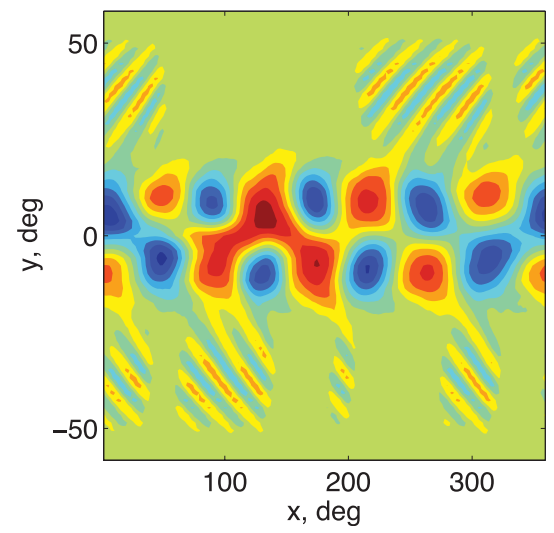

u

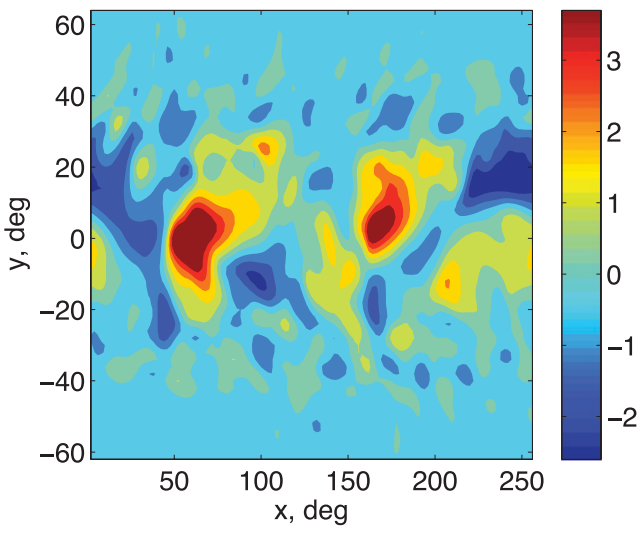

u

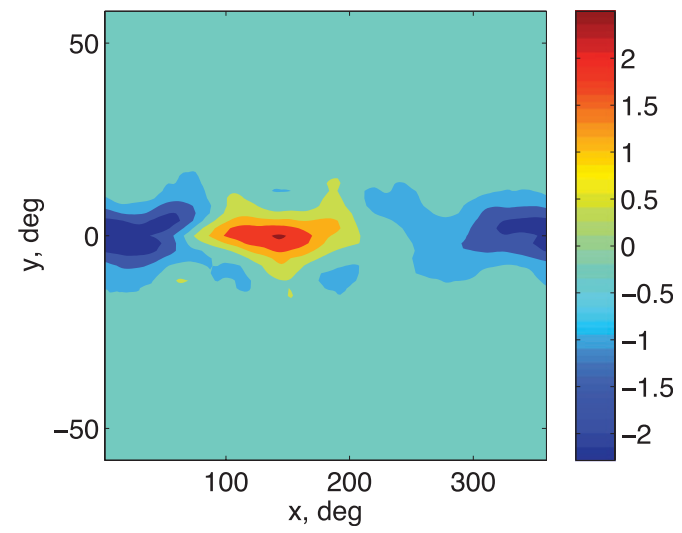

FIG. 7. Snapshots of $u\left(\mathrm{~m} \mathrm{~s}^{-1}\right)$ from the (top) SW and (bottom) QE models, showing (left) a snapshot and (right) a 4-day average eliminating much of the Yanai wave signal from both models and the midlatitude inertia-gravity wave variability showing in the SW model.

$\eta_{Y}$ is wavenumber dependent and proportional to the meridionally antisymmetric product of $y$ and a Gaussian. The Gaussian length scale in (5) can be expressed in terms of the phase speed $c$ :

$$
R_{0}^{2}=\frac{c_{0}}{\beta}
$$

Henceforth, $R_{0}$ and $c_{0}$ will be used to denote properties of free waves in the model, while $c_{g}$ will be used to denote the Yanai wave group speed.

The power series expansion of the WISHE heating term in (4), assuming $U<0$ (mean easterlies) and using $\eta-\eta^{*}=\eta+\left|\eta^{*}\right|\left(\right.$ as $\left.\eta^{*}<0\right)$, is

$$
\begin{aligned}
Q / A & =\left(\eta+\left|\eta^{*}\right|\right) \sqrt{(u+U)^{2}+v^{2}} \\
& =\left|\eta^{*}\right||U|+\eta|U|-\left|\eta^{*}\right| u-\eta u+\frac{\left|\eta^{*}\right|}{2|U|} v^{2}+\cdots
\end{aligned}
$$

This power series formally converges only for small perturbation velocities but we found it to have errors of less than $25 \%$ when compared to the full heating field. We are only concerned with the equatorially symmetric Kelvin response to the Yanai wave contribution to the WISHE forcing. Since the Yanai $u_{Y}$ and $\eta_{Y}$ are equatorially antisymmetric, their product will be symmetric and so will the contribution of $v_{Y}^{2}$. Averaging over a period of the Yanai waves, and noting that the Yanai wave forcing amplitude moves at $c_{g}$, we find an expression for the part of $Q$ that is due to the Yanai wave forcing, which we denote $\langle G\rangle$ :

$$
\begin{aligned}
\langle G\rangle & =A\left(\frac{\left|\eta^{*}\right|}{2|U|}\left\langle v_{Y}^{2}\right\rangle-\left\langle\eta_{Y} u_{Y}\right\rangle\right) \\
& =\hat{v}_{Y}^{2} A e^{-\left(y^{2} / 2 R_{1}^{2}\right)}\left(\frac{\left|\eta^{*}\right|}{2|U|}-\frac{2 \omega^{2} g}{c^{2}} y^{2}\right) e^{i k\left(x-c_{g} t\right)} \\
& =\bar{G}(y) e^{i k\left(x-c_{g} t\right)} .
\end{aligned}
$$

Here $\hat{v}_{Y}^{2}$ is the squared amplitude of Yanai waves. The Yanai forcing depends on the square of the free Yanai wave Gaussian structure and therefore has a narrower meridional structure. We have used 


$$
\left[\exp \left(\frac{-y^{2}}{2 R_{0}^{2}}\right)\right]^{2}=\exp \left(\frac{-2 y^{2}}{2 R_{0}^{2}}\right)=\exp \left(\frac{-y^{2}}{2 R_{1}^{2}}\right)
$$

and have accordingly introduced $R_{1}$ as a new, narrower, meridional length scale:

$$
R_{1}=\frac{1}{\sqrt{2}} R_{0}
$$

Note that the meridional structure of $\langle G\rangle$ would be a Gaussian with scale $R_{1}$ if only the $v$ field of the Yanai group influenced the heating, but the meridional width is further reduced by the additional term $\left\langle\eta_{Y} u_{Y}\right\rangle$, which effectively multiplies the structure by the parabola $\left(1-y^{2}\right)$. We will see below that the narrower meridional structure is consistent with the slower propagation speed of the forced signal.

Given the findings of the previous section that the slow signal shares many characteristics with a Kelvin wave, we look for a forced Kelvin wave response to the surface flux heating induced by the above Yanai group forcing. Setting $v=0$ and using the above Yanai wave forcing $\langle G\rangle$ as well as the linear parts of the WISHE term induced by the forced Kelvin wave response,

$$
\begin{aligned}
u_{t} & =-\eta_{x}, \\
\beta y u & =-\eta_{y}, \\
\eta_{t}+H u_{x} & =-\bar{G}(y) e^{i k\left(x-c_{g} t\right)}-A\left(|U| \eta+\left|\eta^{*}\right| u\right)-\frac{\eta}{\tau} .
\end{aligned}
$$

The right-hand side of (13) contains the Yanai group forcing $\langle G\rangle$, two terms from the power series expansion for the heating induced by the Kelvin wave response, and the Newtonian damping. A linear response must be propagating at the speed of the forcing, so we look for a solution for the slowly propagating signal of the form

$$
(u, \eta)=e^{-\left(y^{2} / 2 R_{G}^{2}\right)} e^{i k\left(x-c_{g} t\right)}\left(u_{G}, \eta_{G}\right),
$$

with some as yet unspecified meridional scale $R_{G}$. Inserting (14) into (11) and (12) gives

$$
\begin{aligned}
c_{g} u_{G} & =\eta_{G}, \\
\beta R_{G}^{2} u_{G} & =\eta_{G} .
\end{aligned}
$$

The consistency of these two equations requires that $c_{g}=\beta R_{G}^{2}<c_{0} / 2$. Without this specific relation, the response cannot take the assumed form of a forced Kelvin wave. If the Yanai group heating depended only on $v_{Y}^{2}$, then $c_{g}=c_{0} / 2$, and it is simple to see that $R_{G}=R_{1}$, which is the scale of the squared Yanai Gaussian. The condition $c_{g}=c_{0} / 2$ is satisfied exactly for a Yanai wave of $k=0$, as can be derived from its dispersion relation (see, e.g., Gill 1982). This describes the situation for our SW model. The fact that the meridional width of the Yanai heating is further reduced by its dependence on $u$ and $\eta$ of the Yanai waves, as for example is the case for our QE model, renders $c_{0} / 2$ an upper bound for the speed of the forced Kelvin wave response. More generally, the meridional scale of the slow signal is consistent with its speed of propagation, which is equal to that of the Yanai wave group acting as the forcing. As long as the meridional structure of the WISHE forcing projects on that of the slow signal, the excitation of the slow signal is possible.

Equation (13) can be used together with (15) to find the relationship between the response and the forcing:

$$
\frac{G}{u_{G}}=-i k\left(c_{0}^{2}-c_{g}^{2}\right)+A\left(\left|\eta^{*}\right|+c_{g}|U|\right)-\frac{c_{g}}{\tau} .
$$

In the simulations shown in the previous section, the terms $A c_{g}|U|-c_{g} / \tau$ are almost an order of magnitude smaller than the other real terms, so we may write

$$
u_{G} \approx G \frac{A\left|\eta^{*}\right|+i k\left(c_{0}^{2}-c_{g}^{2}\right)}{\left(A\left|\eta^{*}\right|\right)^{2}+k^{2}\left(c_{0}^{2}-c_{g}^{2}\right)^{2}} .
$$

The wavenumber dependence of the response indicates that the amplitude of the response decreases with wavenumber, consistent with the spectra presented earlier (Fig. 3) and with observations of the MJO. The phase of the slow signal response relative to the WISHE heating is also predicted by this expression, as will be discussed below.

To summarize the proposed mechanism: the Yanai waves are destabilized by the WISHE term and tend to be westward propagating in the presence of a nonzero convective delay time (Emanuel 1993). The Yanai waves contribute to a WISHE heating that is symmetric about the equator via both the $\left\langle v_{Y}^{2}\right\rangle$ and $\left\langle u_{Y} \eta_{Y}\right\rangle$ terms, which leads to an envelope of Yanai heating propagating at the Yanai group speed (8). The meridional structure of the Yanai forcing is of a modified Gaussian, narrower than the equatorial Rossby radius of deformation. This Yanai envelope creates a forced Kelvin wave response (seen in the Hovmöller plot of Fig. 1) that propagates at the same speed as the Yanai envelope and has a 
meridional structure that is narrower than that of the free Kelvin waves. The forced Kelvin wave excitation is especially effective when its narrower meridional structure projects well on the modified Gaussian structure of the Yanai forcing.

The Kelvin wave response does not linearly feed back into the Yanai waves because the equatorially symmetric Kelvin structure does not project onto the equatorially antisymmetric $u$ and $\eta$ fields of the Yanai waves. Nonlinear interactions may still occur and might be indicated by some features of the model power spectra (Fig. 3).

To further investigate the meridional structure of the slow signal, we applied a filter in wavenumber-frequency space to isolate the variability in the region below the free Kelvin dispersion curve. Figure 8 shows the meridional structure of the zonal mean of the root time-mean square of the filtered zonal velocity anomalies. The profile appears similar to a Gaussian but is narrower than the Gaussian profile of a free Kelvin wave, which is also plotted as $\exp \left[-y^{2} /\left(2 R_{0}^{2}\right)\right]$. In the case of the shallow water model, the profile of the rms variability of the slow signal is very close to the square of the Gaussian structure of the Yanai $v$ velocity (or, equivalently, of the Kelvin $u$ and $\eta$ fields). This seems to strongly support the above mechanism for the slowly propagating anomalies, and to suggest that the $\left\langle u_{Y} \eta_{Y}\right\rangle$ term does not considerably alter the heating in that model.

The rms variability in the shallow water model remains significant at higher latitudes, consistent with the higher off-equatorial inertial gravity wave variability in that model. The slow signal in the QE model has a narrower structure than the square of the Gaussian, which is consistent with the fact that the signal seems to propagate more slowly in that model.

\section{c. Phase between Yanai group heating and westerly response}

It is worth noting that the above nonlinear WISHE mechanism seen in the QE model produces an eastwardpropagating disturbance, in mean easterlies, with peak surface heat fluxes that are just slightly west of the peak convective activity. This is dramatically different from linear WISHE theories that produce peak surface heat fluxes east of the convection (Emanuel 1987; Neelin et al. 1987) and is, incidentally, qualitatively consistent with the phasing in the observed MJO.

The phase relation between dynamic and thermodynamic variables in the slow signal provides additional evidence for the mechanism discussed above and helps in understanding its energetics. As noted previously, the zonal wind and height fields of the slow signal are in phase, with negligible meridional wind, all of which is consistent with the dynamical structure of a Kelvin wave.
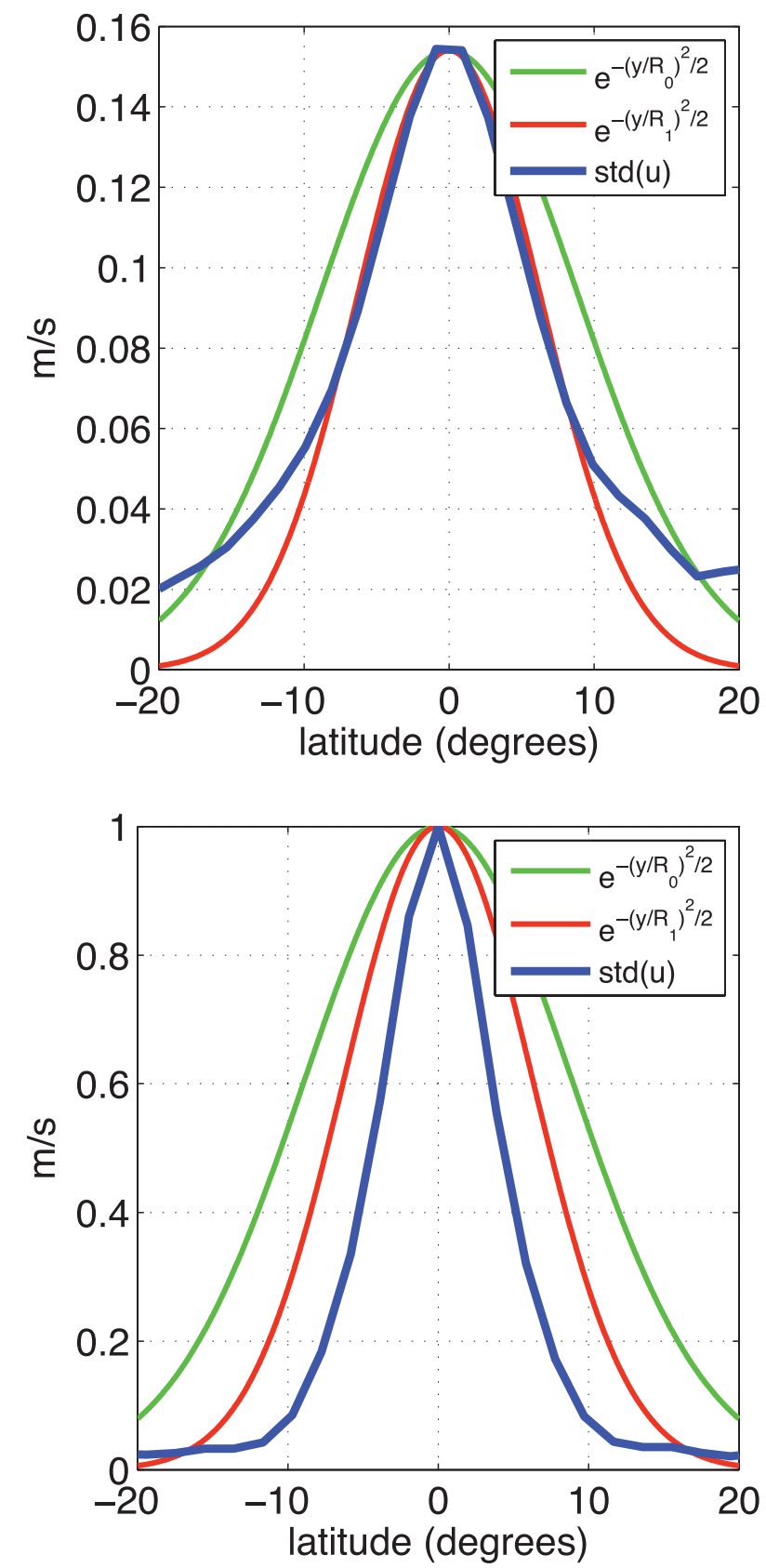

FIG. 8. Rms of the filtered $u\left(\mathrm{~m} \mathrm{~s}^{-1}\right)$ averaged over longitude (blue lines), and Gaussian distributions having length scales $R_{0}$ (green lines) and $R_{1}$ (red lines) as defined in the text, for the (top) SW and (bottom) QE models.

We now examine the phase relation between this Kelvin wave structure and various components of the heating field in the two models.

The relation (17) derived above gives the phase relation between the Yanai group forcing and the Kelvin wave response. We find that the real part of its right-hand side is positive and larger than the imaginary part, showing 
that $G$ is nearly in phase with the westerlies of the forced Kelvin response. This is consistent with the phasing of $v^{2}$ and $u$ shown in Fig. 5, where $u$ lags $v^{2}$ by only a small amount.

The net heating by surface fluxes is $A\left(\left|\eta^{*}\right|+|U| c_{g}\right) u_{G}-$ $G$, so that the phase of this heating with $u$ is given by

$$
\frac{A\left(\left|\eta^{*}\right|+|U| c_{g}\right) u_{G}-G}{u_{G}}=i k\left(c_{0}^{2}-c_{g}^{2}\right)+\frac{c_{g}}{\tau} .
$$

The real part of the right-hand side is the radiative damping term, which is found to have a magnitude smaller than that of the imaginary term for low zonal wavenumbers. For a response of zonal wavenumber 3 , which is roughly the scale of the positive anomalies seen in Fig. 5, this amounts to the heating leading $u$ by slightly less than $90^{\circ}$. This is consistent with Fig. $5 \mathrm{~b}$, although the somewhat skewed distribution of $u$ and $Q$ in longitude makes it difficult to precisely assess phase relationships in this plot.

Physically, this means that the Yanai group forcing, due to the $\left\langle v^{2}\right\rangle$ component of the heating, is balanced by the sum of radiative damping and surface fluxes induced by the Kelvin response. In a Kelvin wave in background easterlies, because $u$ is in phase with $\eta$, the Newtonian damping has a diabatic effect that augments the Yanai group forcing, and the sum of these components is opposed by the Kelvin-induced WISHE heating. In other words, the WISHE heating induced by the Kelvin response must overcompensate for the Yanai group heating, feeding energy into the forced Kelvin wave that is dissipated by the Newtonian damping. This energetic description is consistent with the positive correlation between $\langle-Q\rangle$ and $\langle\eta\rangle$ seen in Fig. 5b. In addition, a calculation of terms in the continuity equation reveals that the WISHE term and the Newtonian cooling term do roughly balance each other.

Although the Newtonian damping might thus seem to play a central role in the energetics of the slow signal in the shallow water model, the absence of Newtonian damping in the QE model shows that the existence of the slow signal does not require this damping. The QE model employs a constant radiative cooling, so that the Newtonian damping term is absent in the analogous version of (19) for that model. Interpretation of the energetics in the QE model is complicated by the fact that the actual heating term in that model is the convective mass flux $M$, which is mostly balanced by the adiabatic cooling due to ascent; only a small residual is influenced by the surface heat flux $E$, and $E$ is furthermore decoupled from $M$ in regions of strong subsidence. These facts are all consistent with the structure seen in
Fig. 6c, in that $\langle M\rangle$ in is largest where there is convergence, and thus ascent, and $M$ also seems to have a component in phase with $\langle E\rangle$.

\section{d. Sensitivity to model parameters}

The existence of the slowly propagating signal in the two different models considered here, together with the consistency of the general mechanism in these models, indicates that the proposed mechanism is robust to model formulation and parameters.

A comprehensive sensitivity analysis of the shallow water model was described by Solodoch (2010), demonstrating robustness to model parameters, boundary conditions, functional form and spatial extent of the heating, etc. In general, we find that the slow signal and its mechanism are robust in a range of spatial and temporal numerical resolutions and in a range of model parameter values in both models. The signal is not produced if mean westerlies are prescribed, which we argue is because Yanai waves are not destabilized in such a background state. We discuss alternative mechanisms for exciting the Yanai waves in the absence of easterlies in section $4 \mathrm{e}$.

As noted in section 2, the slow signal was produced in the QE model for low values of the parameter $\chi$, which controls the degree to which downdrafts dry and cool the subcloud layer. The values of $\chi$ needed to generate this signal were sufficiently low that subcloud-layer relative humidity in the QE model was typically near 95\%, producing a mean state with net surface heat fluxes about an order of magnitude lower than in observations. At higher values of $\chi$, which produced lower subcloud relative humidities and higher surface heat fluxes, tropical variability was dominated by free Kelvin waves and only very weak power was distributed near the theoretical Yanai dispersion curve. While it is possible that the very humid limit in which the QE model was integrated for this study renders it unrealistic and the mechanism irrelevant to observations, it is also possible that the slow signal would appear in a more realistic part of parameter space if a strong Yanai wave group could be generated there. While controls on the relative amplitude of different equatorial wave types merit further exploration, it is entirely possible that those controls would involve mechanisms largely independent of what we have proposed to couple Yanai wave and sub-Kelvin variability (e.g., Andersen and Kuang 2008).

Observations of convectively coupled tropical waves (Wheeler and Kiladis 1999) indicate that the equivalent depth for the tropical atmosphere is between 10 and $50 \mathrm{~m}$. It is simple to see that if we had changed the prescribed model equivalent depth from $50 \mathrm{~m}$ to, say, $30 \mathrm{~m}$, this would slow all of the waves participating in the proposed mechanism, including the forced Kelvin 
waves comprising the slowly propagating signal. But one does not expect the mechanism to qualitatively change.

\section{e. Discussion and relevance to observed intraseasonal variability}

While some features of the slowly propagating signal are reminiscent of the observed $\mathrm{MJO}$, we emphasize that there are important differences as well. Even if this signal and mechanism do not explain specific observed features, they are interesting from a theoretical point of view and may add to our understanding of tropical dynamics.

The propagation speed of the signal is similar to that of the MJO, especially in the QE model with its convective delay that produces slower Yanai waves. The Kelvin structure of the westerlies and their phase with the convective heating again match the observations. However, the composites from both models, as well as the snapshots, clearly show that the slowly propagating signal is not accompanied by Rossby gyres as is typical of the observations (Kiladis et al. 2005), thereby weakening the case for this mechanism as an explanation of the observed MJO. We also note that the convectively coupled Yanai wave variance peaks over the tropical central Pacific (e.g., Hendon and Liebmann 1991; Wheeler and Kiladis 1999), while the MJO variance is stronger over the Indian Ocean and the western Pacific. Similarly, in our model simulations meridional winds due to Yanai waves dominate the instantaneous picture, while westerlies and westerly wind bursts seem to dominate in observations.

In the present simplified models, the Yanai waves are destabilized through (linear) WISHE in the presence of mean easterlies because easterlies occur in the warm phase of these waves, producing a positive correlation between WISHE-induced convective heating and temperature. This explains why the existence of the slow propagating signal depends on mean easterlies. In cloud-resolving model simulations, more detailed conceptual models, and likely in the real atmosphere as well, Yanai waves (and convectively coupled waves in general) can be excited in the absence of mean easterlies through stratiform instability and/or moisture-stratiform instability (e.g., Mapes 2000; Kuang 2008a,b). These instability mechanisms are discussed in detail in the cited references and arise because convective heating in the upper troposphere is relatively weakly controlled by temperature anomalies in the upper troposphere and more strongly controlled by both temperature and moisture anomalies in the lower troposphere. This allows for collocation of positive convective heating anomalies and positive temperature anomalies, leading to an instability (Kuang 2010).

The spectra shown in Figs. 3 and 4 share some features with the observed ones, in particular the presence of an eastward-propagating signal under the dispersion curve of the free Kelvin wave and some power near the Yanai wave dispersion curve. However, these spectra are in general different from observations in some key points. First, the observed MJO spectrum tends to have peak power at a constant frequency, so that $d \omega / d k \approx 0$. Our solutions do not show this characteristic. The spectrum of the QE model also has some large-amplitude sharp features (e.g., the crosslike spectral maximum in the $v$ spectrum; Fig. 4) that are very different from the observed spectrum. In spite of these unrealistic features, spectra from the QE model have a fairly weak red-noise background and, unlike spectra from the shallow water model, contain little energy in the high-frequency inertiagravity wave region (Solodoch 2010). In some ways the QE model thus provides a cleaner result, making it clear that the inertia-gravity waves are not part of the mechanism driving the slow signal.

The disagreement with some key observed features makes it clear that the mechanism of the slowly propagating signal analyzed here cannot be considered in its present form a complete explanation of the MJO. This mechanism may turn out to be relevant to other observed intraseasonal variability or to the variability of aquaplanet simulations, but more work is required to examine these possibilities.

\section{Conclusions}

Two intermediate complexity models were used to explore large-scale organization of the tropical atmosphere in the presence of nonlinear WISHE effects. One is a shallow water model, and the other is a model of the tropical atmosphere's first baroclinic mode, with an additional prognostic equation for boundary layer moisture and a quasi-equilibrium treatment of moist convection. With both models, a slowly eastward-propagating signal appears robustly in the experiments. This signal propagates at $\sim 10 \mathrm{~m} \mathrm{~s}^{-1}$ or about half the speed of a linear Kelvin wave in the shallow water model, while in the QE model the propagation speed is $\sim 5 \mathrm{~m} \mathrm{~s}^{-1}$.

The slow eastward-propagating signal was shown to be a forced Kelvin wave with a meridional spatial structure consistent with an equivalent depth smaller than that specified in the model. The signal is also coherent with a group of long Yanai waves that has a group velocity similar to that of the slow forced Kelvin wave. We showed that the Yanai group nonlinearly forces the slow Kelvin-like signal through the nonlinear WISHE term, and explained how the nonlinear WISHE term leads to the smaller effective equivalent depth and slow propagation of the signal. 
The suggested mechanism predicts the slower Kelvin wave meridional structure and speed. The excited signal in the numerical simulations is strongest at $k=1-3$, again consistent with the suggested mechanism. The phenomenon has a multiscale character in space and time, and an interesting and novel aspect of the mechanism proposed here is the critical role of higher-frequency motions involving the Yanai waves. Concurrently with Solodoch (2010), Solodoch et al. (2010), and the present study, Yang and Ingersoll $(2009,2011)$ have presented an idea that is related in terms of linking the MJO to the Yanai waves, even if without the nonlinear WISHE forcing mechanism proposed here.

Although it is clearly premature to propose that the mechanism found here is applicable to the MJO or to any other observed intraseasonal phenomenon, it is worth noting that the forced Kelvin wave signal identified here shares some features with the MJO. The forced Kelvin waves do propagate at speeds significantly slower than the moist Kelvin wave speeds and have spectral power peaks at the $k=1$ and 2 wavenumbers; additionally, there is asymmetry in zonal wind toward higher westerlies than easterlies, particularly close to the convection center. Unlike the linear WISHE modes of Emanuel (1987) and Neelin et al. (1987), surface evaporation here peaks west of the peak convective heating, resembling the observations. There are also notable differences. For example, the present mechanism appears to operate in our models only in mean easterlies. However, there is nothing in our hypothesis relating Yanai wave to subKelvin variability that requires mean easterlies. The mean easterlies are needed in these models only to destabilize the Yanai waves, and an alternative mechanism for producing Yanai waves may lead to the same slow signal found here. Such an alternative mechanism may be the destabilization of Yanai waves by a stratiform instability in the absence of mean easterlies (Mapes 2000; Kuang 2008b), as discussed above, making the proposed mechanism potentially relevant in areas such as the Indian and western Pacific Oceans. Such issues clearly require further study. These issues notwithstanding, we feel the nonlinear WISHE mechanism that we have identified in this paper is an interesting and nontrivial mechanism that can enrich our understanding of tropical dynamics. It is interesting to also note that observations do show significant power in tropical, convectively coupled Yanai waves, which play a central role in our mechanism (Pires et al. 1997; Wheeler and Kiladis 1999).

Acknowledgments. This work is based on Aviv Solodoch's M.A. thesis at the Weizmann Institute. We thank Harry Hendon and two anonymous reviewers for their helpful comments. We also thank the three reviewers and editors of Geophysical Research Letters for their comments on an earlier draft of this manuscript. ET and ZK are supported by the NSF climate dynamics program, Grant ATM-0754332. WRB was supported by a Daly Postdoctoral Fellowship in the Department of Earth and Planetary Sciences at Harvard, and by the French Environmental Fellowship at the Harvard University Center for the Environment. ET thanks the Weizmann Institute for its hospitality during parts of this work.

\section{APPENDIX}

\section{The Quasi-Equilibrium Model}

The reader is directed to Emanuel $(1987,1993)$ for more detailed information on the thermodynamic assumptions of the model, which are described only briefly here.

We assume that convection keeps variations in $s^{*}$, the saturation moist entropy of the free troposphere, constant with height. Then using the hydrostatic relation and one of Maxwell's relations, variations in the geopotential $\Phi$ can be partitioned into a barotropic component $\delta \Phi_{0}$ that is invariant with height and a baroclinic component that can be written in terms of variations in $s^{*}$ :

$$
\frac{\partial}{\partial p} \delta \Phi=-\left(\frac{\partial T}{\partial p}\right)_{s^{*}} \delta s^{*}+\frac{\partial}{\partial p} \delta \Phi_{0},
$$

where the derivative of $T$ is taken at constant $s^{*}$. In this particular model we assume no variations in surface pressure, so that the above can be integrated to obtain $\delta \Phi$ :

$$
\delta \Phi-\delta \Phi_{b}=\left(T_{b}-T\right) \delta s^{*},
$$

where the $b$ subscript denotes a property at the top of the subcloud layer and $T$ serves as a vertical coordinate. Using (A2) together with the property that the vertical integral of purely baroclinic $\Phi$ perturbations must vanish gives

$$
-\delta \Phi=(T-\bar{T}) \delta s^{*}
$$

where $\bar{T}$ is a mass-weighted vertical mean tropospheric temperature.

Conservation equations for horizontal winds, linearized about a mean zonal wind $U$ and phrased on an equatorial $\beta$ plane, can then be written in terms of the fluctuating component of $s^{*}$ (the $\delta$ symbol is henceforth omitted): 


$$
\begin{aligned}
& \frac{\partial u}{\partial t}+U \frac{\partial u}{\partial x}-\beta y v=(T-\bar{T}) \frac{\partial s^{*}}{\partial x}-\frac{\partial}{\partial x} \Phi_{0}+F_{u} \\
& \frac{\partial v}{\partial t}+U \frac{\partial v}{\partial x}+\beta y u=(T-\bar{T}) \frac{\partial s^{*}}{\partial y}-\frac{\partial}{\partial y} \Phi_{0}+F_{v}
\end{aligned}
$$

with the $F$ terms representing both surface drag and diffusion.

We now write conservation equations for $s^{*}$ and the subcloud-layer entropy $s_{b}$, choosing to linearize the advective part of the conservation equations for $s^{*}$ and $s_{b}$ while preserving the positive-definite nature of the convective mass flux:

$$
\begin{aligned}
\frac{\partial s^{*}}{\partial t}+U \frac{\partial s^{*}}{\partial x} & =-N^{2}\left(w-\epsilon M_{c}\right)-R, \\
H_{b}\left(\frac{\partial s_{b}}{\partial t}+U \frac{\partial s_{b}}{\partial x}\right) & =E+\min \left[0,\left(w_{b}-M_{c}\right)\right]\left(s_{b}-s_{m}\right),
\end{aligned}
$$

where $M_{c}$ is the net upward mass flux in convective clouds, $H_{b}$ is the depth of the subcloud layer, $w_{b}$ is the vertical velocity at the top of the subcloud layer averaged over clear and cloudy areas, $\epsilon$ is bulk precipitation efficiency, $E$ is the surface entropy flux, and $R$ is the rate of radiative cooling, which is fixed at $1 \mathrm{~K} \mathrm{day}^{-1}$. The second term on the right-hand side of the $s_{b}$ equation represents the downward advection of low-entropy midtropospheric air into the boundary layer, with the difference between $s_{b}$ and the midtropospheric entropy $s_{m}$ hereafter assumed to be a constant, denoted $\chi$. The min function is used to eliminate this term when the net mass flux is upward. A dry static stability is defined:

$$
N^{2} \equiv c_{p} \Delta T \frac{\Gamma_{d}}{\Gamma_{m}} \frac{\partial \ln \theta}{\partial z},
$$

with $\Gamma_{d}$ and $\Gamma_{m}$ being the dry and moist adiabatic temperature lapse rates, respectively. The surface entropy flux is given by a bulk transfer formula:

$$
E=C_{k}|\mathbf{V}|\left(s_{o}^{*}-s_{b}\right),
$$

where $s_{o}^{*}$ is the saturation moist entropy at the temperature of the sea surface. Here $|\mathbf{V}|$ is the total surface wind speed, including the imposed basic-state wind $U$.

Subcloud-layer equilibrium is used to specify an equilibrium cloud-base mass flux:

$$
M_{\mathrm{eq}}=w+\frac{1}{\chi}\left[E-H_{b} U \frac{\partial s_{b}}{\partial x}\right] .
$$

The actual convective mass flux is relaxed toward this equilibrium value over a time scale of $3 \mathrm{~h}$ and is constrained to be nonnegative. We also set $M_{c}=0$ if $s_{b}<s^{*}$, so that there is no convection where the atmosphere is stable.

\section{Modal decomposition}

The horizontal wind is projected onto a single baroclinic mode $\mathbf{v}_{1}$ that uses temperature as a vertical coordinate:

$$
\mathbf{v} \equiv T^{\prime} \mathbf{v}_{1}
$$

where $T^{\prime}$ is the temperature anomaly normalized by $\Delta T \equiv T_{s}-\bar{T}:$

$$
T^{\prime} \equiv \frac{T-\bar{T}}{\Delta T}
$$

No dynamical boundary layer is used in the momentum equations, and $u_{1}$ and $v_{1}$ can be taken to represent baroclinic winds at the surface.

With these assumptions, the vertical velocity must have a vertical structure independent of horizontal position and time,

$$
w(x, y, z, t)=\bar{w}(x, y, t) \Omega(z),
$$

with the vertical structure obtained from the temperature profile:

$$
\Omega(z)=\int_{0}^{z} T^{\prime} d z
$$

In this framework, continuity is then

$$
\bar{w}=-\frac{\partial u_{1}}{\partial x}-\frac{\partial v_{1}}{\partial y} .
$$

Prognostic equations for $u_{1}$ and $v_{1}$ are obtained by substituting (A9) into (A4), multiplying by $T^{\prime}$, and integrating from the surface to the tropopause:

$$
\begin{aligned}
& \frac{\partial u_{1}}{\partial t}+U \frac{\partial u_{1}}{\partial x}=\Delta T \frac{\partial s^{*}}{\partial x}+\beta y v_{1}-\frac{C_{D}}{\left\langle T^{\prime 2}\right\rangle H}|\mathbf{V}| u_{1}, \\
& \frac{\partial v_{1}}{\partial t}+U \frac{\partial v_{1}}{\partial x}=\Delta T \frac{\partial s^{*}}{\partial y}-\beta y u_{1}-\frac{C_{D}}{\left\langle T^{\prime 2}\right\rangle H}|\mathbf{V}| v_{1},
\end{aligned}
$$

where angle brackets represent mass-weighted vertical integrals, which are represented in pressure coordinates for simplicity. Quantities on the left-hand side are the time tendency and advective terms. Quantities on the right-hand side are the pressure gradient terms (phrased in terms of $s^{*}$ ) and the Coriolis, drag, and horizontal diffusion terms. The drag terms have been written using 
TABLE A1. Parameters used in the QE model.

\begin{tabular}{|c|c|c|}
\hline Symbol & Value & Description \\
\hline$\Delta T$ & $30 \mathrm{~K}$ & $\begin{array}{l}\text { Surface minus mean } \\
\text { atmospheric temperature }\end{array}$ \\
\hline$H_{b}$ & $910 \mathrm{~m}$ & Mean boundary layer depth \\
\hline$H$ & $14 \mathrm{~km}$ & Mean tropopause height \\
\hline$N^{2}$ & $1.55 \mathrm{~J} \mathrm{~kg}^{-1} \mathrm{~m}^{-1}$ & Dry static stability \\
\hline$\left\langle T^{\prime 2}\right\rangle$ & 0.57 & $\begin{array}{l}\text { Normalized second moment } \\
\text { of temperature }\end{array}$ \\
\hline$\langle\Omega\rangle$ & $1.6 \times 103 \mathrm{~m}$ & Vertical mean structure function \\
\hline$\Omega_{\mathrm{b}}$ & $788 \mathrm{~m}$ & $\begin{array}{l}\text { Structure function at top of } \\
\text { subcloud layer }\end{array}$ \\
\hline$C_{D}$ & 0.001 & $\begin{array}{l}\text { Surface transfer coefficient } \\
\text { for momentum }\end{array}$ \\
\hline$C_{k}$ & 0.001 & Enthalpy exchange coefficient \\
\hline$R$ & $1.5 \mathrm{~K} \mathrm{day}^{-1}$ & Radiative cooling rate \\
\hline$\epsilon$ & 0.8 & Bulk precipitation efficiency \\
\hline$\theta_{e b}-\theta_{m}$ & $6 \mathrm{~K}$ & $\begin{array}{l}\text { Entropy drop at top of } \\
\text { subcloud layer }\end{array}$ \\
\hline \multirow[t]{2}{*}{$\tau_{c}$} & $3 \mathrm{~h}$ & Convective response time \\
\hline & $28^{\circ} \mathrm{C}$ & Sea surface temperature \\
\hline
\end{tabular}

a bulk flux formula for momentum. The depth of the troposphere $H$ is assumed constant.

The conservation equation for $s^{*}$, given in (A5), must also be vertically integrated to eliminate the heightdependence of $w$ and $M_{c}$ :

$$
\frac{\partial s^{*}}{\partial t}=-N^{2}\langle\Omega\rangle\left(\bar{w}-\epsilon \bar{M}_{c}\right)-R+\kappa \frac{\partial^{2} s^{*}}{\partial y^{2}},
$$

where $R$ is assumed to be a prescribed, vertically uniform constant. The horizontal advection terms have been eliminated by the assumption that $s^{*}$ is constant with height. The conservation equation for $s_{b}$ does not need to be modified, although care must be taken to use the values of $w$ and $M_{c}$ at the top of the subcloud layer and the mean value of $v$ within that layer:

$$
H_{b}\left(\frac{\partial s_{b}}{\partial t}+U \frac{\partial s_{b}}{\partial x}\right)=E+\min \left[0,\left(\bar{w}-\bar{M}_{c}\right)\right] \Omega_{b} \chi .
$$

Here $\Omega_{b}$ is the value of $\Omega$ at the top of the subcloud layer, and $v_{b}$ is the mass-weighted meridional wind vertically integrated within the subcloud layer. The equilibrium mass flux then becomes

$$
\bar{M}_{\mathrm{eq}}=\bar{w}+\frac{1}{\chi \Omega_{b}}\left(E-H_{b} U \frac{\partial s_{b}}{\partial x}\right),
$$

with $\bar{M}_{c}$ relaxed toward $\bar{M}_{\text {eq }}$ over a 6-h time scale.

The model consists of (A6), (A7), (A10), and (A13)(A17), which include the time tendency equations for the prognostic variables $s^{*}, s_{b}, u_{1}$, and $v_{1}$. The parameters dependent on temperature were derived assuming a surface temperature of $296 \mathrm{~K}$ at $1000 \mathrm{hPa}$, a dry adiabat up to $900 \mathrm{hPa}$, and a moist pseudoadiabat from 900 to $150 \mathrm{hPa}$. The values used for model parameters are listed in Table A1.

\section{REFERENCES}

Andersen, J., and Z. Kuang, 2008: A toy model of the instability in the equatorially trapped convectively coupled waves on the equatorial beta plane. J. Atmos. Sci., 65, 3736-3757.

Boos, W. R., and K. A. Emanuel, 2008: Wind-evaporation feedback and abrupt seasonal transitions of weak, axisymmetric Hadley circulations. J. Atmos. Sci., 65, 2194-2214.

Emanuel, K. A., 1987: An air-sea interaction model of intraseasonal oscillations in the tropics. J. Atmos. Sci., 44, 2324 2340.

_ 1993: The effect of convective response time on WISHE modes. J. Atmos. Sci., 50, 1763-1776.

_ tions in convecting atmospheres. Quart. J. Roy. Meteor. Soc., 120, 1111-1143.

Gill, A. E., 1980: Some simple solutions for heat-induced tropical circulation. Quart. J. Roy. Meteor. Soc., 106, 447-462. , 1982: Atmosphere-Ocean Dynamics. Academic Press, 662 pp.

Hendon, H., and B. Liebmann, 1991: The structure and annual variation of antisymmetric fluctuations of tropical convection and their association with Rossby-gravity waves. J. Atmos. Sci., 48, 2127-2140.

Kiladis, G. N., K. H. Straub, and P. T. Haertel, 2005: Zonal and vertical structure of the Madden-Julian oscillation. J. Atmos. Sci., 62, 2790-2809.

Kuang, Z., 2008a: Modeling the interaction between cumulus convection and linear gravity waves using a limited-domain cloud system-resolving model. J. Atmos. Sci., 65, 576-591.

_ 2008b: A moisture-stratiform instability for convectively coupled waves. J. Atmos. Sci., 65, 834-854.

- 2010: Linear response functions of a cumulus ensemble to temperature and moisture perturbations and implications for the dynamics of convectively coupled waves. J. Atmos. Sci., 67, 941-962.

Lindzen, R. S., and S. Nigam, 1987: On the role of sea surface temperature gradients in forcing low-level winds and convergence in the tropics. J. Atmos. Sci., 44, 2418-2436.

Madden, R. A., and P. R. Julian, 1971: Detection of a 40-50-day oscillation in the zonal wind in tropical Pacific. J. Atmos. Sci., 28, 702-708.

—, and —-1994: Observations of the 40-50-day tropical oscillation-A review. Mon. Wea. Rev., 122, 814-837.

Majda, A., and S. Stechmann, 2009: The skeleton of tropical intraseasonal oscillations. Proc. Natl. Acad. Sci. USA, 106, $8417-8422$.

Maloney, E. D., and A. H. Sobel, 2004: Surface fluxes and ocean coupling in the tropical intraseasonal oscillation. J. Climate, 17, 4368-4386.

Mapes, B. E., 2000: Convective inhibition, subgrid-scale triggering energy, and stratiform instability in a toy tropical wave model. J. Atmos. Sci., 57, 1515-1535.

Marshall, J., A. Adcroft, C. Hill, L. Perelman, and C. Heisey, 1997a: A finite-volume, incompressible Navier Stokes model for studies of the ocean on parallel computers. J. Geophys. Res., 102 (C3), 5753-5766. 
, C. Hill, L. Perelman, and A. Adcroft, 1997b: Hydrostatic, quasi-hydrostatic, and nonhydrostatic ocean modeling. J. Geophys. Res., 102 (C3), 5733-5752.

Neelin, J. D., and J.-Y. Yu, 1994: Modes of tropical variability under convective adjustment and the Madden-Julian oscillation. Part I: Analytical theory. J. Atmos. Sci., 51, 18761894.

—_, and N. Zeng, 2000: A quasi-equilibrium tropical circulation model-Formulation. J. Atmos. Sci., 57, 1741-1766.

— I. M. Held, and K. H. Cook, 1987: Evaporation-wind feedback and low-frequency variability in the tropical atmosphere. J. Atmos. Sci., 44, 2341-2348.

Pires, P., J.-L. Redelsperger, and J.-P. Lafore, 1997: Equatorial atmospheric waves and their association to convection. Mon. Wea. Rev., 125, 1167-1184.

Raymond, D. J., 2001: A new model of the Madden-Julian oscillation. J. Atmos. Sci., 58, 2807-2819.

Shinoda, T., H. H. Hendon, and J. Glick, 1998: Intraseasonal variability of surface fluxes and sea surface temperature in the tropical western Pacific and Indian Oceans. J. Climate, 11, 1685-1702.

Sobel, A. H., E. D. Maloney, G. Bellon, and D. Frierson, 2008: The role of surface heat fluxes in tropical intraseasonal oscillations. Nat. Geosci., 1, 653-657.

Solodoch, A., 2010: Excitation of slow Kelvin waves in the equatorial atmosphere by Yanai wave-group-induced convection. M.S. thesis, Weizmann Institute of Science, 85 pp.
— W. Boos, Z. Kuang, and E. Tziperman, cited 2010: Excitation of slow MJO-like Kelvin waves in the equatorial atmosphere by Yanai wave-group via a WISHE-induced convection. [Available online at http://arxiv.org/abs/1002.2340.]

Straub, K. H., and G. N. Kiladis, 2003: The observed structure of convectively coupled Kelvin waves: Comparison with simple models of coupled wave instability. J. Atmos. Sci., 60, 1655-1668.

Sugiyama, M., 2009a: The moisture mode in the quasi-equilibrium tropical circulation model. Part I: Analysis based on the weak temperature gradient approximation. J. Atmos. Sci., 66, 1507-1523. , 2009b: The moisture mode in the quasi-equilibrium tropical circulation model. Part II: Nonlinear behavior on an equatorial $\beta$ plane. J. Atmos. Sci., 66, 1525-1542.

Wang, B., 1988: Comments on "An air-sea interaction model of intraseasonal oscillations in the tropics." J. Atmos. Sci., 45, 3521-3525.

Wheeler, M., and G. N. Kiladis, 1999: Convectively coupled equatorial waves: Analysis of clouds and temperature in the wavenumber-frequency domain. J. Atmos. Sci., 56, 374-399.

Yang, D., and A. P. Ingersoll, 2009: Testing a Rossby-wave theory of the MJO. Eos, Trans. Amer. Geophys. Union, 90 (Fall Meeting Suppl.), Abstract A51F-0180.

— and - 2011: Testing the hypothesis that the MJO is a mixed Rossby-Gravity wave packet. J. Atmos. Sci., 68, 226-239.

Zhang, C., 2005: Madden-Julian oscillation. Rev. Geophys., 43, RG2003, doi:10.1029/2004RG000158. 